\title{
Two sampling methods yield distinct microbial signatures in the nasopharynges of asthmatic children
}

\author{
Marcos Pérez-Losada ${ }^{1,2,3^{*}}$, Keith A. Crandall ${ }^{1}$ and Robert J. Freishtat ${ }^{2}$
}

\begin{abstract}
Background: The nasopharynx is a reservoir for pathogens associated with respiratory illnesses, such as asthma. Next-generation sequencing (NGS) has been used to characterize the nasopharyngeal microbiome during health and disease. Most studies so far have surveyed the nasopharynx as a whole; however, less is known about spatial variation (biogeography) in nasal microenvironments and how sampling techniques may capture that microbial diversity.
\end{abstract}

Findings: We used targeted 165 rRNA MiSeq sequencing and two different sampling strategies [nasal washes (NW) and nasal brushes (NB)] to characterize the nasopharyngeal microbiota in 30 asthmatic children. Nasal brushing is more abrasive than nasal washing and targeted the inner portion of the inferior turbinate. This region is expected to be different from other nasal microenvironments. Nasal washing is not spatially specific. Our $30 \times 2$ nasal microbiomes generated 1,474,497 sequences, from which we identified an average of 157 and 186 OTUs per sample in the NW and NB groups, respectively. Microbiotas from NB showed significantly higher alpha-diversity than microbiotas from NW. Similarly, both nasal microbiotas were distinct from each other (PCOA) and significantly differed in their community composition and abundance in at least 9 genera (effective size $\geq 1 \%$ ).

Conclusions: Nasopharyngeal microenvironments in asthmatic children contain microbiotas with different diversity and structure. Nasal washes and brushes capture that diversity differently. Future microbial studies of the nasopharynx need to be aware of potential spatial variation (biogeography).

Keywords: $16 \mathrm{~S}$ rRNA, Asthma, Microbiome, Microenvironment, Nasopharynx

\section{Findings \\ Background}

The nasopharynx is considered an anatomical reservoir from which pathogenic microbes can spread to the lower and upper respiratory airways and cause respiratory infections [1-3]. Culture-independent sequencing methods have shown that some pathogenic bacterial genera associated with asthma (e.g., Moraxella, Streptococcus, Haemophilus, Neisseria, and Staphylococcus) are also present in the nasopharynx $[1,4-6]$. Consequently, several recent metataxonomic and metagenomic (see [7] for distinction)

\footnotetext{
*Correspondence: mlosada323@gmail.com

${ }^{1}$ Computational Biology Institute, George Washington University, Innovation

Hall, Suite 305, 45085 University Drive, Ashburn, VA 20147, USA

2Division of Emergency Medicine, Children's National Medical Center,

Washington, DC 20010, USA

Full list of author information is available at the end of the article
}

studies have investigated how nasopharyngeal microbial communities change during health and disease in relation to clinical variation $[1,3,5,8-16]$. All these nextgeneration sequencing (NGS) studies, however, either sampled the nasopharynx as a whole or focused on a particular anatomical area (microenvironment); hence, less is known about the spatial variation (biogeography) in microbial composition of the nasopharynx. The nose has a complicated and diverse anatomical structure, comprised of diverse epithelial cells and glands with different physiologies and functions $[17,18]$. Hence, it seems reasonable to expect that different microenvironments in the nose will also harbor distinct microbial communities. However, to our knowledge, only one study has assessed the biogeography of the nasal microbiota [19]. In that study, the authors used 16S rRNA sequence data to compare the microbiotas of three nasal sites (anterior naris, middle meatus, and 
sphenoethmoidal recess) in healthy subjects and detected significant differences in diversity between the anterior nares and the two inner mucosal sites. No study, so far, has investigated the biogeography of the nasal cavity in asthmatic patients.

In this report, we used targeted $16 \mathrm{~S}$ rRNA sequencing and two different sampling techniques (nasal washes and nasal brushes) to characterize the nasopharyngeal microbiota in asthmatic children. Nasal brushing is more abrasive than nasal washing and was used to target a particular region of the nasopharynx, the inner portion of the inferior turbinate; nasal washing is assumed to be less spatially specific and to reach the main cavities in the nasopharynx. Hence, given these differences in sampling methodology, we hypothesize that nasal microbiotas collected by nasal washes will be different in alpha- and betadiversity from those collected by nasal brushes.

\section{Methods}

\section{Ethics approval and consent to participate}

All participants in this study were part of the AsthMaP2 (Asthma Severity Modifying Polymorphisms) Study [20]. AsthMaP2 is an ongoing study of urban children and adolescents designed to find associations among airway microbes, environmental exposures, allergic sensitivities, genetics, and asthma. AsthMaP2 and the study presented here were approved by the Children's National Medical Center Institutional Review Board (Children's National IRB), which requires that consent is obtained and documented prior to conducting study procedures and collection of samples for research. Written consent was obtained from all independent participants or their legal guardians using the Children's National IRB approved informed consent documents (IRB No PRO00002517).

\section{Samples and molecular analyses}

A total of 30 children and adolescents (ages 6 to 17 years) were recruited from the metropolitan Washington, DC, area. All had been physician-diagnosed with asthma for at least one year prior to recruitment. Individuals who reported a medical history of chronic or complex cardiorespiratory disease were ineligible. Their nasopharynges were simultaneously sampled using both nasal washes (NW) and nasal brushes (NB). NW samples were procured by instilling $5 \mathrm{ml}$ of isotonic sterile saline buffer into each nare, holding it for $10 \mathrm{~s}$, and then blowing into a specimen collection container. NB samples were procured by brushing the mucosa of the inner section of the inferior turbinate of each nostril with a sterile nylon bristle cytology brush (CytoSoft Cytology Brush No. CYB-1, Medical Packaging Corporation Camarillo, CA). That mucosa is covered with a mucus blanket that traps smaller particulate matter and bacteria [21]. Moreover, cytology brushes are designed to harvest epithelial cells, meaning
NB sampling is generally more abrasive than NW sampling, and so, more likely to collect the microorganisms closely attached to the nasal mucosa. Nonetheless, since the cytology brushes are not protected, we cannot ensure that these samples represent the inferior turbinate microbial community alone, and it is possible that upon removal of the brush from the sinuses, unintended mucus, hair, and epithelial cells from the nares were also collected. Nasal washes are non-specific and may target a different microbial population than nasal brushes, which will pick up microbes attached to the mucosal surface instead of non-attached microbes along the sinonasal cavity (including the inferior turbinate).

Total DNA was extracted using the QIAGEN QIAamp DNA Kit (Catalog \# 51304). Before adding the ATL buffer, samples were pre-incubated in $100 \mathrm{uL}$ of lysozyme-TE buffer $\mathrm{pH}=8.0$ for $30 \mathrm{~min}$ at $37{ }^{\circ} \mathrm{C}$. All extractions yielding $>50 \mathrm{ng}$ of total DNA (as indicated by NanoDrop 2000 UV-vis Spectrophotometer measuring) were further processed. DNA extractions were prepared for sequencing using the Schloss' MiSeq_WetLab_SOP protocol (09.2015) in Kozich et al. [22]. Each DNA sample was amplified for the V4 region ( $250 \mathrm{bp})$ of the $16 \mathrm{~S}$ rRNA gene, and libraries were sequenced using the Illumina MiSeq sequencing platform at University of Michigan Medical School.

\section{Sequence analyses}

Raw FASTQ files were processed in mothur v1.35.1 [23]. Default settings were used to minimize sequencing errors as described in Schloss et al. [24]. Clean sequences were aligned to the SILVA_v123 bacterial reference alignment at www.mothur.org. Chimeras were removed using uchime [25], and non-chimeric sequences were classified using the naïve Bayesian classifier of Wang et al. [26]. Sequences were clustered into Operational Taxonomic Units (OTUs) at the 0.03 threshold (species level). OTU sequence representatives and taxonomy were imported (BIOM format) into QIIME [27] for subsequent analyses. The mothur OTU table was filtered to a minimum of two observations (sequences) per OTU. Samples were subsampled (rarefaction analysis) to the smallest sample size ( 1,100 sequences) to remove the effect of sample size bias on community composition. Nonetheless, OTU differential abundance tests that take advantage of full sample sizes (metagenomeSeq zeroinflated Gaussian [28]) were also applied for comparison.

Trees for phylogenetic diversity calculations were constructed using FastTree [29]. Taxonomic alpha-diversity was estimated as the number of observed OTUs and by the Chao1, Simpson, and Shannon indexes. Phylogenetic alphadiversity (PD) was calculated by the Faith's phylogenetic diversity index [30]. Similarly, both taxonomic (Bray-Curtis and Euclidean) and phylogenetic (unweighted and weighted UniFrac) beta-diversity metrics were calculated. Community 


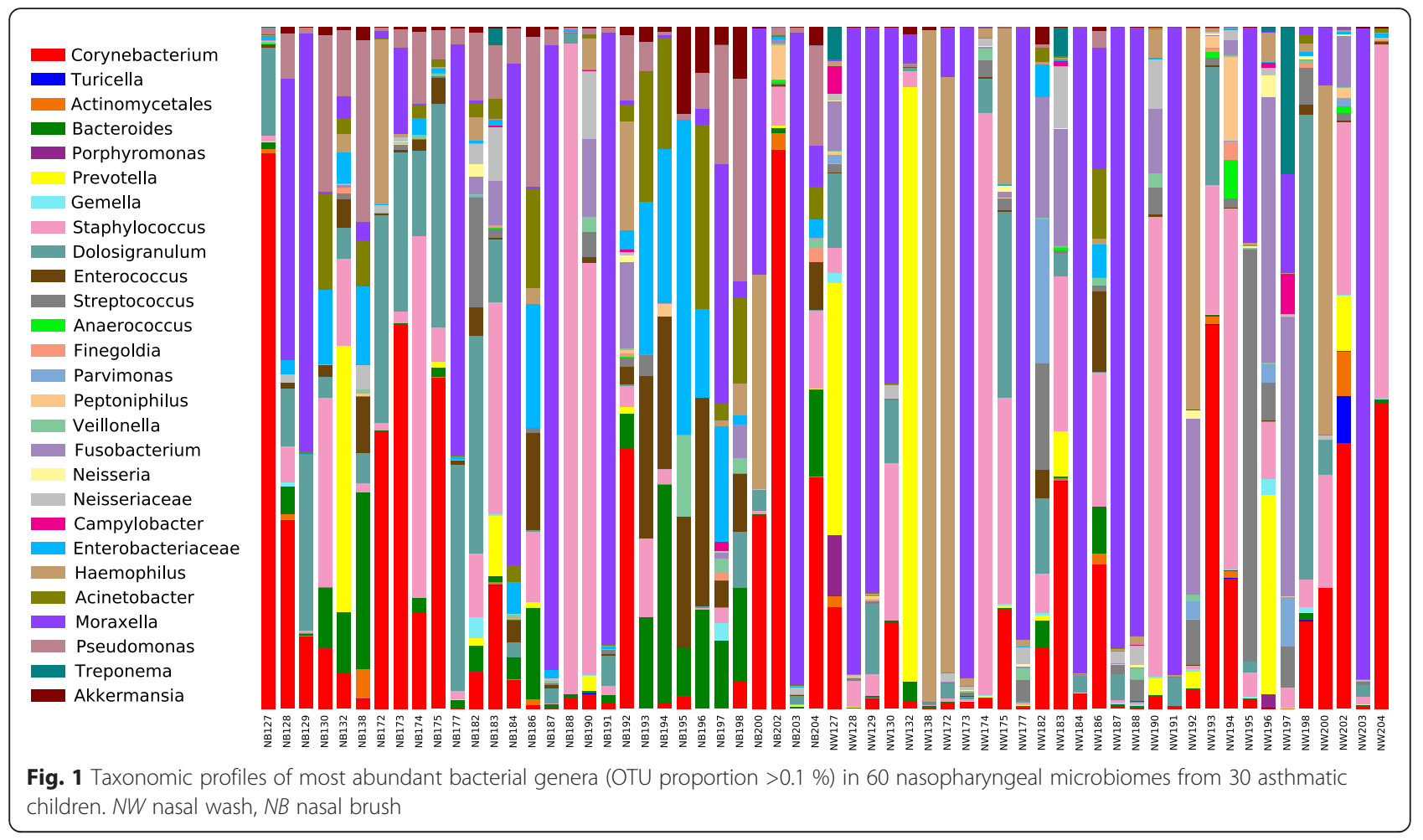

dissimilarity was estimated using principal coordinates analysis ( $\mathrm{PCoA})$. Alpha- and beta-diversity metrics were compared between samples grouped by sampling strategy (NW versus $\mathrm{NB}$ ) using both parametric and non-parametric versions of the $t$ test. Taxonomic and phylogenetic distances were also compared among those groups using the nonparametric PERMANOVA test from the vegan R's library [31]. Significance was determined through 10,000 permutations. Finally, OTU abundance differences between NW and NB groups and between paired samples (e.g., NW patient 1 versus NB patient 1) were assessed using rarefied (White's non-parametric $t$ test [32] and Fisher's exact test, respectively) and non-rarefied (metagenomeSeq zero-inflated
Gaussian) data. Core microbiome analyses were also performed to identify resident bacteria. Bonferroni or Benjamini-Hochberg FDR multiple test correction methods were applied. All analyses were performed in mothur, QIIME, STAMP [33], and RStudio [34].

\section{Availability of the data and materials}

Sequence data have been uploaded to the GenBank under SRA accession number SRP069020. All materials used for nasal brushing and washing were processed as regular samples using the same QIAGEN DNA purification kit and PCR protocols. No PCR band was visible on an agarose gel.

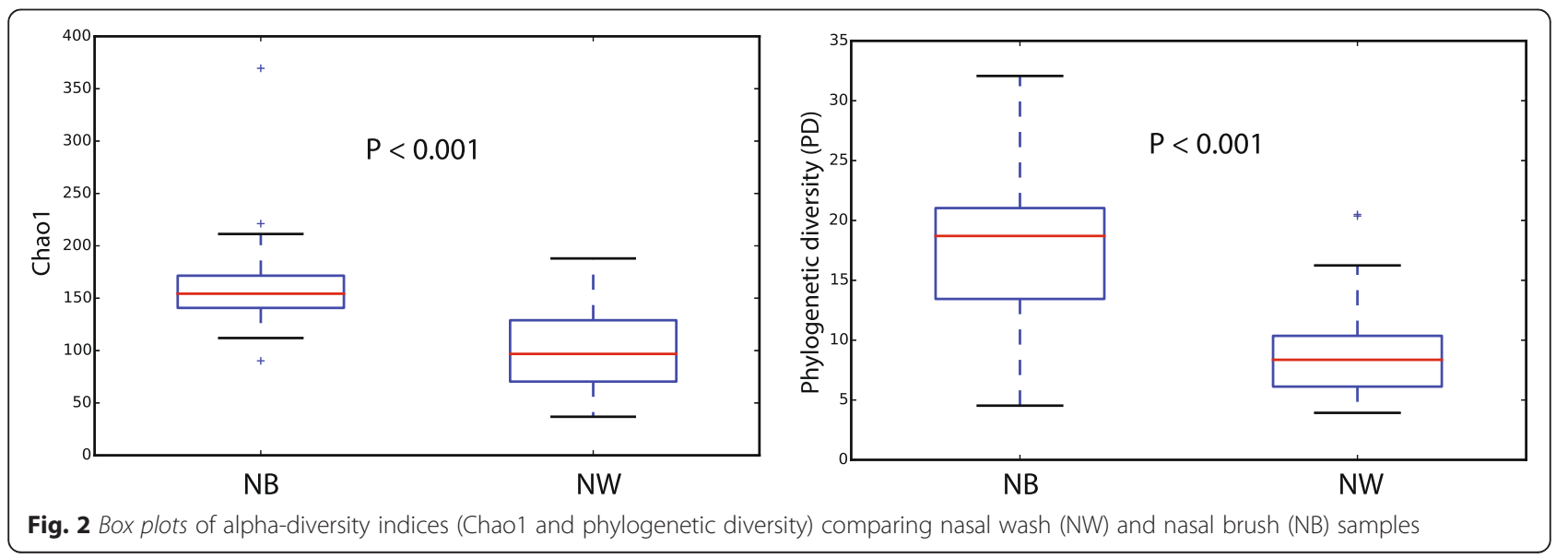




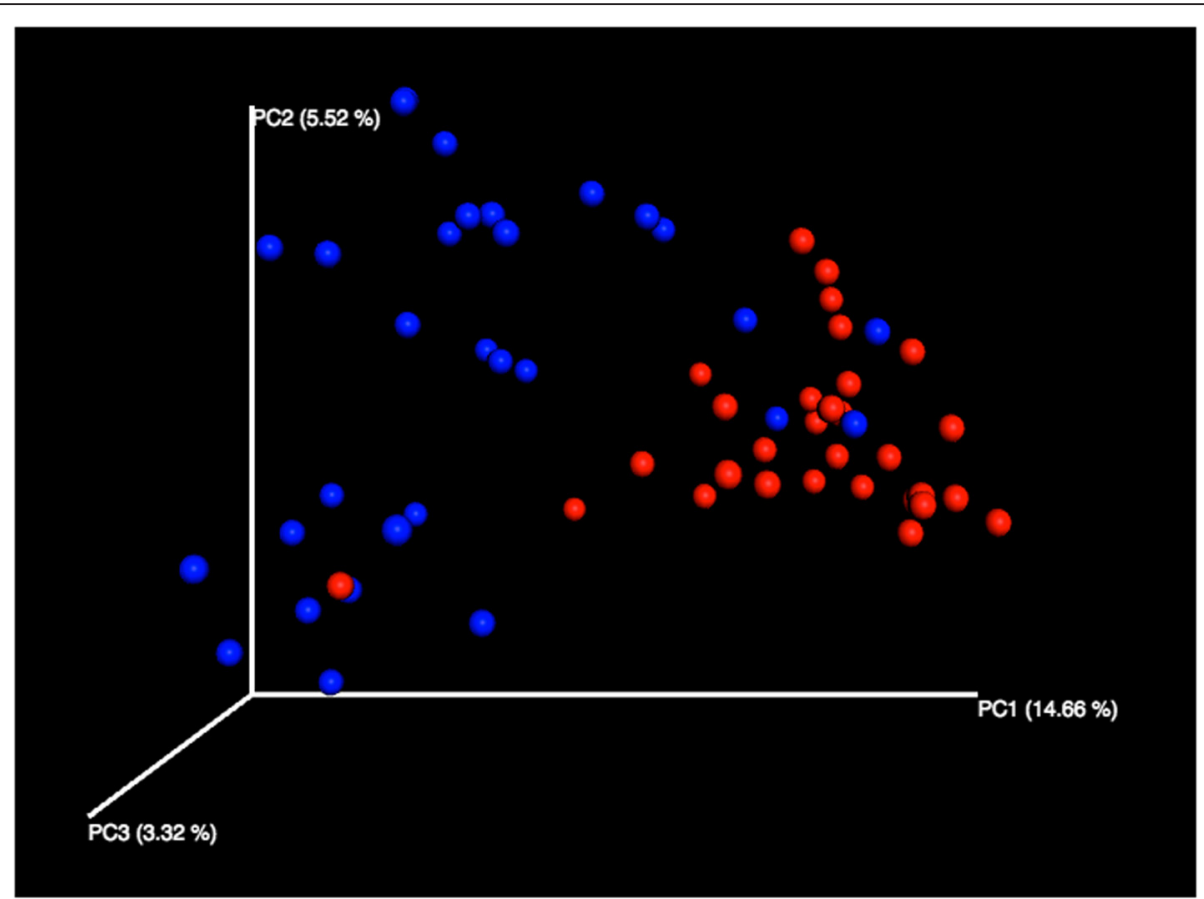

Fig. 3 3D principal coordinates analysis (unweighted UniFrac distances) of nasal wash (NW) and nasal brush (NB) samples

\section{Results}

\section{Sequences and OTUs}

Sixty nasal microbiomes corresponding to 30 asthmatic children were analyzed via MiSeq sequencing of $16 \mathrm{~S}$ rRNA $\mathrm{V} 4$ amplicons. A total of 1,474,497 sequences ranging from 1100 to 62,148 sequences per sample (mean $=24,575$; median $=20,599$ ) were obtained after quality control analyses and OTU filtering. From these data, we identified 33-272 OTUs (mean $=157$ ) per sample in the NW group and $120-408$ OTUs $($ mean $=186)$ per sample in the NB group.

\section{Microbial composition of the nasopharynx}

Nasopharyngeal microbiomes were dominated by the following seven genera, which showed different proportions in NW and NB groups: Moraxella $(\mathrm{NW}=38.1 \%, \mathrm{NB}=$
$27.8 \%)$, Staphylococcus (NW = $15.4 \%, \quad \mathrm{NB}=10.2 \%)$, Corynebacterium (NW = 8.6 \%, NB = 20.4\%), Haemophilus $(\mathrm{NW}=8.4 \%, \mathrm{NB}=4.2 \%)$, Fusobacterium $(\mathrm{NW}=5.8 \%$, $\mathrm{NB}=1.1 \%)$, Prevotella $(\mathrm{NW}=3.8 \%, \mathrm{NB}=1.1 \%)$, and Dolosigranulum (NW = $3.7 \%$, NB = $8.7 \%$ ) (see Fig. 1). The core microbiome analysis (OTUs present in $95 \%$ of the samples) identified five OTUs of the genera Moraxella, Staphylococcus, Haemophilus, Streptococcus, and Enterococcus in NB and four OTUs of the genera Moraxella, Pseudomonas, Enterococcus, and Bacteroides in NW. This suggests that NW and NB may contain different resident bacteria. All dominant genera reported here for asthmatic children and adolescents have been detected in other microbiome studies of the nasopharynx in infants with and without respiratory infections [1, 3, 16, 35], although in
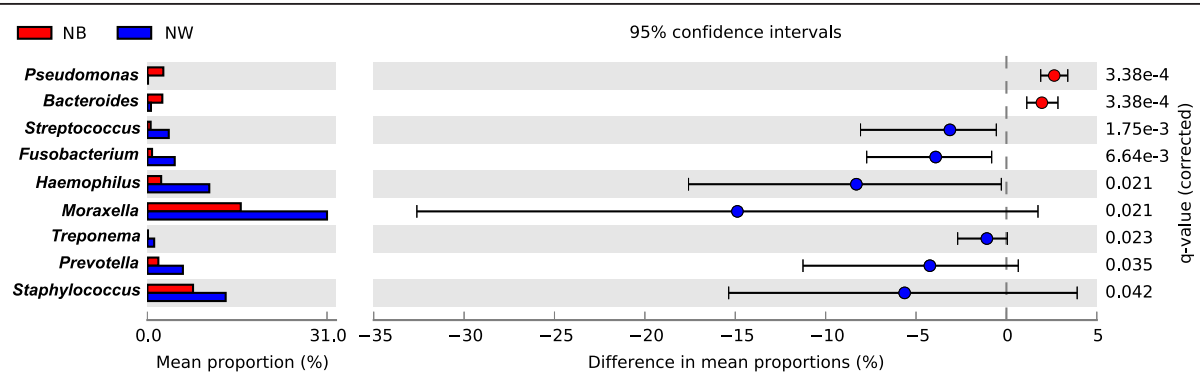

Fig. 4 Extended error bar plot showing the nine bacterial genera with a significant difference (White's test; $P<0.05$ ) in proportions of at least $1 \%$ between nasal wash (NW) and nasal brush (NB) samples. Seven genera are overabundant within the nasal microbiotas collected by washing compared to those collected by brushing 
different proportions. Similarly, microbial profiles observed in our children also looked different from those described in the nasopharynx of adults $[19,36,37]$.

\section{Nasopharyngeal washes and brushes sampled different microbiotas}

Microbial profiles of the most abundant bacterial genera (Fig. 1) and alpha-diversity indices (observed OTUs, Chao1, Shannon, Simpson, and PD) varied greatly between NW and NB groups (Fig. 2), with NB showing greater estimates for all indices. These differences were significant in all cases (non-parametric $t$ test; $P<0.001$ ). A previous NGS $16 \mathrm{~S}$ metataxonomic study of nasopharyngeal microenvironments showed higher alpha-diversity values (same indices as here) in microbiotas from the posterior sections of the nasopharynx (represented by the middle meatus and sphenoethmoidal recess) than in those from the anterior naris [19]. In accordance with their results, we also see a similar trend in microbiotas mainly collected from the inferior turbinate (NB) compared to those non-specifically sampled along the sinonasal cavity (NW).

Beta-diversity ordination analysis of $\mathrm{PCoA}$ showed clear dissimilarities between NW and NB microbiomes for all distances tested (Fig. 3). Similarly, significant differences $\left(P<0.001 ; R^{2}=0.072\right)$ in community composition (PERMANOVA) were observed between both groups after FDR correction. Finally, microbial abundances also significantly varied between NW and NB groups (White's test; effect size $\geq 1 \%$ and metagenomeSeq zero-inflated Gaussian) after FDR correction for the following nine genera: Staphylococcus, Prevotella, Treponema, Streptococcus, Moraxella, Haemophilus, Fusobacterium, Bacteroides, and Pseudomonas (Fig. 4). Similarly, all 30 comparisons of paired samples showed significant differences for 3 to 26 OTUs (Fisher's exact test with an effective size filter of $\geq 1 \%$ ). As above, PCoA analysis in Yan et al. [19] also revealed a separation between anterior nare samples and more posterior mucosal samples. Their ANOVA testing of OTU relative abundances between those two regions detected 18 OTUs that significantly differed between them, although 16 of those relative abundances were $<0.1 \%$. Our analyses showed larger differences in community composition between nasal washes and nasal brushes from potentially different environments in the nasopharynx.

We believe that the observed differences in alpha- and beta-diversity are due to the fact that our brushes tapped on a richer microbial microenvironment in the nasopharynx (inner portion of the inferior turbine) and sampled it more efficiently than the less specific nasal washing. These more diverse microbiotas could represent established bacterial communities in the deeper areas of the nasopharynx, while the less diverse microbiotas sampled by nasal washing would correspond to transient bacterial communities in the nasopharynx.

\section{Conclusions}

With the exception of the vagina, explorations of spatial differences within the human microbiome have been heavily guided by gross anatomical landmarks and boundaries [19]. As a result, microenvironments within otherwise homogenous-appearing human organs, and especially within spatially constrained sites (like the nasopharynx), have been relatively ignored. This report is a first attempt to characterize the nasopharyngeal microbiotas of asthmatic children at a finer spatial scale. It identified distinct microbial signatures in $\mathrm{NB}$ and $\mathrm{NW}$ samples, which may be due to the distinct microenvironments sampled or the sampling approach itself. Future microbial studies need to be aware of the potential for spatial variation (biogeography) in the nasopharynx and choose the best sampling approach to target the nasal areas of interest. Our next goal is to characterize the nasopharyngeal microbiome in more depth by brushing the nasal vestibule and turbinates (inferior, middle, and superior) to distinguish resident from transient bacteria. Then, we will use this information to compare nasal, oral, and tracheal microbiotas, so airway microbial dynamics during asthma can be better understood.

\section{Acknowledgements \\ We thank the GWU Colonial One High Performance Computing Cluster for computational time.}

\section{Authors' contributions}

All authors conceived and designed the study. MP-L and RJF collected samples and sequence data. MP-L analyzed the data and wrote the manuscript with all authors contributing. All authors read and approved the final manuscript.

\section{Competing interests}

The authors declare that they have no competing interests.

\section{Consent for publication}

Written informed consent was obtained from the patient for publication of this case report. A copy of the written consent is available for review by the Editor-in-Chief of this journal.

\section{Author details}

${ }^{1}$ Computational Biology Institute, George Washington University, Innovation Hall, Suite 305, 45085 University Drive, Ashburn, VA 20147, USA. ²Division of Emergency Medicine, Children's National Medical Center, Washington, DC 20010, USA. ${ }^{3} \mathrm{CIBIO}-\mathrm{InBIO}$, Centro de Investigação em Biodiversidade e Recursos Genéticos, Universidade do Porto, Campus Agrário de Vairão, Vairão 4485-661, Portugal.

Received: 11 February 2016 Accepted: 6 May 2016

Published online: 16 June 2016

\footnotetext{
References

1. Teo SM, Mok D, Pham K, Kusel M, Serralha M, Troy N, Holt BJ, Hales BJ, Walker ML, Hollams $\mathrm{E}$, et al. The infant nasopharyngeal microbiome impacts severity of lower respiratory infection and risk of asthma development. Cell Host Microbe. 2015;17:704-15.

2. Bogaert D, De Groot R, Hermans PW. Streptococcus pneumoniae colonisation: the key to pneumococcal disease. Lancet Infect Dis. 2004;4(3):144-54.
} 
3. Biesbroek G, Tsivtsivadze E, Sanders EA, Montijn R, Veenhoven RH, Keijser BJ, Bogaert D. Early respiratory microbiota composition determines bacterial succession patterns and respiratory health in children. Am J Respir Crit Care Med. 2014:190(11):1283-92.

4. Hilty M, Burke C, Pedro H, Cardenas P, Bush A, Bossley C, Davies J, Ervine A, Poulter $L$, Pachter $L$, et al. Disordered microbial communities in asthmatic airways. PLoS One. 2010;5(1):e8578.

5. Pérez-Losada M, Castro-Nallar E, Bendall ML, Freishtat RJ, Crandall KA. Dual transcriptomic profiling of host and microbiota during health and disease in pediatric asthma. PLoS One. 2015:10(6):e0131819.

6. Dickson RP, Erb-Downward JR, Huffnagle GB. The role of the bacterial microbiome in lung disease. Expert Rev Respir Med. 2013;7(3):245-57.

7. Marchesi JR, Ravel J. The vocabulary of microbiome research: a proposal. Microbiome. 2015;3:31

8. Feazel LM, Santorico SA, Robertson CE, Bashraheil M, Scott JA, Frank DN, Hammitt LL. Effects of vaccination with 10-valent pneumococcal nontypeable haemophilus influenza protein D conjugate vaccine (PHiD-CV) on the nasopharyngeal microbiome of Kenyan toddlers. PLoS One. 2015;10(6): e0128064

9. Prevaes SM, de Winter-de Groot KM, Janssens HM, de Steenhuijsen Piters WA, Tramper-Stranders GA, Wyllie AL, Hasrat R, Tiddens HA, van Westreenen $M$, van der Ent CK, et al. Development of the nasopharyngeal microbiota in infants with cystic fibrosis. Am J Respir Crit Care Med. 2016; 193(5):504-15.

10. Cremers AJ, Zomer AL, Gritzfeld JF, Ferwerda G, van Hijum SA, Ferreira DM, Shak JR, Klugman KP, Boekhorst J, Timmerman HM, et al. The adult nasopharyngeal microbiome as a determinant of pneumococcal acquisition. Microbiome. 2014;2:44

11. Allen EK, Koeppel AF, Hendley JO, Turner SD, Winther B, Sale MM. Characterization of the nasopharyngeal microbiota in health and during rhinovirus challenge. Microbiome. 2014;2:22.

12. Biesbroek G, Bosch AA, Wang X, Keijser BJ, Veenhoven RH, Sanders EA, Bogaert D. The impact of breastfeeding on nasopharyngeal microbial communities in infants. Am J Respir Crit Care Med. 2014;190(3):298-308.

13. Sakwinska O, Bastic Schmid V, Berger B, Bruttin A, Keitel K, Lepage M, Moine D, Ngom Bru C, Brussow H, Gervaix A Nasopharyngeal microbiota in healthy children and pneumonia patients. J Clin Microbiol. 2014;52(5):1590-4.

14. Bassis CM, Tang AL, Young VB, Pynnonen MA. The nasal cavity microbiota of healthy adults. Microbiome. 2014;2:27.

15. Castro-Nallar E, Shen Y, Freishtat RJ, Pérez-Losada M, Manimaran S, Liu G, Spira A, Johnson WE, Crandall KA. Integrating metagenomics and host gene expression to characterize asthma-associated microbial communities. BMC Med Genet. 2015;8:50.

16. Bogaert D, Keijser B, Huse S, Rossen J, Veenhoven R, van Gils E, Bruin J, Montijn R, Bonten M, Sanders E. Variability and diversity of nasopharyngeal microbiota in children: a metagenomic analysis. PLoS One. 2011;6(2):e17035.

17. Geurkink N. Nasal anatomy, physiology, and function. J Allergy Clin Immunol. 1983;72(2):123-8.

18. Jones $\mathrm{N}$. The nose and paranasal sinuses physiology and anatomy. Adv Drug Deliv Rev. 2001;51(1-3):5-19.

19. Yan M, Pamp SJ, Fukuyama J, Hwang PH, Cho DY, Holmes S, Relman DA. Nasal microenvironments and interspecific interactions influence nasal microbiota complexity and S. aureus carriage. Cell Host Microbe. 2013;14(6):631-40.

20. Benton AS, Wang Z, Lerner J, Foerster M, Teach SJ, Freishtat RJ. Overcoming heterogeneity in pediatric asthma: tobacco smoke and asthma characteristics within phenotypic clusters in an African American cohort. Asthma. 2010;47(7):728-34.

21. Cohen NA. Sinonasal mucociliary clearance in health and disease. Ann Otol Rhinol Laryngol Suppl. 2006;196:20-6.

22. Kozich JJ, Westcott SL, Baxter NT. Highlander SK, Schloss PD. Development of a dual-index sequencing strategy and curation pipeline for analyzing amplicon sequence data on the MiSeq Illumina sequencing platform. Appl Environ Microbiol. 2013;79(17):5112-20.

23. Schloss PD, Westcott SL, Ryabin T, Hall JR, Hartmann M, Hollister EB, Lesniewski RA, Oakley BB, Parks DH, Robinson CJ, et al. Introducing mothur: open-source, platform-independent, community-supported software for describing and comparing microbial communities. Appl Environ Microb. 2009; $75(23): 7537-41$

24. Schloss PD, Gevers D, Westcott SL. Reducing the effects of PCR amplification and sequencing artifacts on $16 \mathrm{~S}$ rRNA-based studies. PLoS One. 2011;6(12):e27310.
25. Edgar RC, Haas BJ, Clemente JC, Quince C, Knight R. UCHIME improves sensitivity and speed of chimera detection. Bioinformatics. 2011;27(16): 2194-200.

26. Wang Q, Garrity GM, Tiedje JM, Cole JR. Naive Bayesian classifier for rapid assignment of rRNA sequences into the new bacterial taxonomy. Appl Environ Microb. 2007;73(16):5261-7.

27. Caporaso JG, Kuczynski J, Stombaugh J, Bittinger K, Bushman FD, Costello EK, Fierer N, Pena AG, Goodrich JK, Gordon Jl, et al. QIIME allows analysis of highthroughput community sequencing data. Nat Methods. 2010;7(5):335-6.

28. Paulson JN, Stine OC, Bravo HC, Pop M. Differential abundance analysis for microbial marker-gene surveys. Nat Methods. 2013;10(12):1200.

29. Price MN, Dehal PS, Arkin AP. FastTree 2-approximately maximum-likelihood trees for large alignments. PloS One. 2010;5(3):e9490.

30. Faith DP. Conservation evaluation and phylogenetic diversity. Biol Conserv. 1992:61:1-10.

31. Dixon P. VEGAN, a package of R functions for community ecology. J Veg Sci. 2003;14(6):927-30.

32. White JR, Nagarajan N, Pop M. Statistical methods for detecting differentially abundant features in clinical metagenomic samples. Plos Comput Biol. 2009; 5(4):e1000352.

33. Parks DH, Tyson GW, Hugenholtz P, Beiko RG. STAMP: statistical analysis of taxonomic and functional profiles. Bioinformatics. 2014;30(21):3123-4.

34. RStudioTeam. RStudio: integrated development for R. Boston, MA: RStudio, Inc; 2016. URL http://www.rstudio.com/.

35. Biesbroek G, Wang X, Keijser BJ, Eijkemans RM, Trzcinski K, Rots NY, Veenhoven RH, Sanders EA, Bogaert D. Seven-valent pneumococcal conjugate vaccine and nasopharyngeal microbiota in healthy children. Emerg Infect Dis. 2014;20(2):201-10,

36. Cremers AJH, Zomer AL, Gritzfeld JF, Ferwerda G, van Hijum SAFT, Ferreira DM, Shak JR, Klugman KP, Boekhorst J, Timmerman HM, et al. The adult nasopharyngeal microbiome as a determinant of pneumococcal acquisition Microbiome. 2014;2:44

37. Bassis CM, Erb-Downward JR, Dickson RP, Freeman CM, Schmidt TM, Young VB, Beck JM, Curtis JL, Huffnagle GB. Analysis of the upper respiratory tract microbiotas as the source of the lung and gastric microbiotas in healthy individuals. mBio. 2015;6(2)::00037.

\section{Submit your next manuscript to BioMed Central and we will help you at every step:}

- We accept pre-submission inquiries

- Our selector tool helps you to find the most relevant journal

- We provide round the clock customer support

- Convenient online submission

- Thorough peer review

- Inclusion in PubMed and all major indexing services

- Maximum visibility for your research

Submit your manuscript at www.biomedcentral.com/submit 